\title{
Numerical software for the inverse estimation of the temperature-dependent thermal properties in heat conduction
}

\author{
J. Zueco \& G. Monreal \\ Universidad Politécnica de Cartagena, ETSII, \\ Departamento de Ingeniería Térmica y Fluidos, Cartagena, Spain
}

\begin{abstract}
A software application has been developed for the numerical solution of inverse problems in heat conduction. The developed software allows the estimation of specific heat and thermal conductivity both individually and simultaneously temperature dependent - and allows application of a variety of boundary conditions. The first step is to accurately estimate the starting point, then estimate the thermophysical properties, presenting the solution by a linear function of straight stretches. The input data is the temperature history at a particular location of the solid. The iterative least-squares approach is used to minimize the functional and in all cases a piecewise function is used to approximate the solution. No prior information is used for the functional forms of the unknown specific heat, because this problem is considered a function estimation problem. In all the cases a piece-wise function is used to approximate the solution. The Network Simulation Method is the numerical method used, with a design of the network model made easy, with very few electric devices. The software developed is used to run the network so that no mathematical manipulations are required. The results confirm that it is possible to estimate the thermophysical properties using experimental temperature history and a procedure inverse. As regards the convergence of the solutions, no restrictions concerning the time interval need to be imposed by the user.
\end{abstract}

Keywords: network model, inverse problem, non-linear thermal processing, temperature dependent thermal properties. 


\section{Introduction}

The estimation of the thermal properties (thermal conductivity and specific heat) from a set of measured temperature data, which is a kind of inverse heat conduction problem (IHCP), is considered in this work. In most practical applications, the thermal conductivity and the specific heat are two functions of temperature and as a result both dependences become non-linear. The development of the inverse problem has progressed. Its resolution permits the determination of more than one thermo-physical property and then to characterize complex materials.

There are many types of inverse problems in heat conduction. An extensive bibliography which includes references to the exact and approximated methods commonly used to solve this kind of problem may be found in Beck et al. [1]. Some are cited here: Osman and Beck [2] estimated the heat transfer coefficient for the cooling of a sphere using the function estimation technique in conjunction with the Sequential Function Specification Method (SFSM). Kim et al. [3] estimated the temperature-dependent thermal conductivity without internal measurements. Sawaf et al. [4] have determined both temperature dependent heat capacity and conductivity using different types of numerical methods. Dantas and Orlande [5] made simultaneous estimations of $\mathrm{k}$ and $\mathrm{C}$ applying the conjugate gradient method; including a study of the influence of sensor location. Carciofi et al. [6] determined the effective thermal diffusivity of mortadeLla from cooking process data. Sacadura and Osman [7] developed a method for to estimate the temperature-dependent emissivity in opaque surfaces indirectly, now that firstly is obtained the superficial temperature and radiative heat net flux. Huang and Özisik [8] obtained precise estimations in a 1-D solid for the case of linear and sinusoidal dependencies of both properties using an approximate direct integration method.

To solve the inverse problem, input data (generally the real temperatures measured by a sensor), are necessary. In the literature these data, taken at a point of the medium at discrete regular (or irregular) times, come from the solution of the direct problem using a random error to simulate real measurements. The direct problem was also solved in this work using NSM.

The Network Simulation Method (NSM, hereafter), which is the numerical technique used to solve both the direct and inverse problem, only requires finitedifference schemes for the spatial variable (as in the lines method) when designing the network model. This method has already been successfully used to solve linear and nonlinear problems in direct (Alhama and González-Fernández [9] and Zueco et al. [10]) and inverse problems. Also, Alhama et al. [11] applied this method to estimating different types of time-dependent incident heat fluxes to verify the general methodology proposed for this inverse heat transfer problem, while Zueco et al. [12] used it to estimate the temperature-dependent thermal properties of solids within the phase-change temperature range. 


\section{Direct and inverse problem}

\subsection{Direct problem}

The set of equations that defines the mathematical model is

$$
\begin{array}{cc}
\rho c_{e} \frac{\partial T}{\partial t}=\frac{\partial}{\partial x}\left(k(T) \frac{\partial T}{\partial x}\right) \quad \text { at } 0<\mathrm{x}<\mathrm{L} \\
-k(T) \frac{\partial T}{\partial t}=q_{0} \quad \text { at } \mathrm{x}=0 \\
-k(T) \frac{\partial T}{\partial t}=0 \quad \text { at } \mathrm{x}=\mathrm{L} \\
\mathrm{T}=\mathrm{To} \quad \text { at } \mathrm{t}=0,0<\mathrm{x}<\mathrm{L}
\end{array}
$$

where $\rho$ is the density, $c_{\mathrm{e}}$ the specific heat and $\mathrm{k}$ the thermal conductivity, $x$ is the position, $t$ the time and $q_{0}$ is the constant heat flux. Eq. (1) is the heat conduction equation. The dimensionless form for these equations is not possible due to the temperature dependence of $k$. Eqs. $(2,3)$ are the constant heat flux and adiabatic boundary conditions at $x=0$ and $x=L$, respectively. Equation (4) gives the initial condition.

\subsection{Inverse problem}

Knowing the solid geometry and physical properties, as the boundary and initial conditions, enables one to solve Eqs. (1-4), thus determining the transient temperature distribution in different points of the solid material. This type of problem is called a direct problem. If any of these magnitudes or a combination of them is unknown, but experimental data are available on the temperature measured inside and/or on the external surface of the solid, one has an inverse problem that allows one to determine the unknown magnitudes, provided those data contain sufficient information.

For the inverse problem of interest here, the parameter is regarded as unknown quantity. For the estimation of such parameter, we consider available the transient temperature measurements $T\left(x_{f}, t_{j}, \varepsilon_{j}\right)=T_{\mathrm{ex}}\left(x_{f}, t_{j}\right)+\omega_{j} \sigma$, where $T_{\mathrm{ex}}\left(x_{f}, t_{j}\right)$ is the temperature solution of the DHCP at the point of the solid $x_{f}$ (where $t_{j}$ is a series of $p$ discrete times within the unsteady period, with $\Delta t$ the time interval between measurements), $\omega_{j}$ is the random number with normal distribution and $\sigma$ is the standard deviation of the errors.

These temperatures were taken at node right, $\mathrm{x}=\mathrm{L}$ (adiabatic condition) or more points. The superscript $\mathrm{j}$ above refers to the time when the measurements are taken. Thus, in this work is desired to minimize the difference between 
experimental and predicted temperatures. Mathematically, it is desired to minimize the objective function,

$$
F\left[x_{\mathrm{f}}, k, \Delta T_{\mathrm{a}}, \Delta t, \varepsilon, z\right]=\Sigma\left[T_{i n v}\left(x_{f}, t_{j}, k\right)-T\left(x_{f}, t_{j}, \varepsilon_{j}\right)\right]^{2}
$$

where $\mathrm{Z}=1,2, \ldots \mathrm{Z}$ identifies the stretch (where $\mathrm{Z}$ is the total number of stretches of the piece-wise function), $k$ the chosen value of the estimated parameter, $\Delta T_{\mathrm{a}}$ the temperature range in each stretch of the estimation (a constant prefixed value). $T_{\text {inv }}\left(x_{f}, t_{j}, k\right)$ is the temperature solution of the inverse problem at position $x_{f}$ and time $t_{j}$. The temperatures $T\left(x_{f}, t_{j}, \varepsilon_{j}\right)$ were defined in eq. (5).

\subsection{Network simulation method}

The starting point is the set of ordinary finite-differential equations, one for each control volume, obtained by spatial discretization of the equation (1). Time remains as a continuous variable in the discretized equations. Based on these equations, a network model is designed, whose equations are formally equivalent to the discretized ones. The variables heat flux $(j)$ and temperature $(T)$ are equivalent to the variables electric current $(J)$ and voltage $(V)$ in this equivalence. A number of networks are connected in series to make up the whole medium and boundary conditions are added by means of special electrical devices.

Firstly, we will summarise the steps used in designing the network circuit of the direct problem. Using Fourier Law, the spatial discretization of eq. (1) gives

$$
\rho c_{\mathrm{e}} \mathrm{d} T_{\mathrm{i}} / \mathrm{d} t=2 k_{\mathrm{i}-\Delta x}\left(T_{\mathrm{i}-\Delta x}-T_{\mathrm{i}}\right) / \Delta x^{2}-2 k_{\mathrm{i}+\Delta x}\left(T_{\mathrm{i}}-T_{\mathrm{i}+\Delta x}\right) / \Delta x^{2}
$$

where $k_{\mathrm{i} \pm \Delta \mathrm{x}}$, are the conductivities in both ends of the volume element (cell) i, which depends on the temperatures $T_{\mathrm{i} \pm \Delta x}$. Eq. (6), written as $j_{\mathrm{i}}-j_{\mathrm{i}-\Delta \mathrm{x}}+j_{\mathrm{i}+\Delta \mathrm{x}}=0$, where

$$
\begin{gathered}
\mathrm{j}_{\mathrm{i}}=\rho \text { ce } \mathrm{dT}_{\mathrm{i}} / \mathrm{dt} \\
\mathrm{j}_{\mathrm{i}+\Delta \mathrm{x}}=\left(\mathrm{T}_{\mathrm{i}}-\mathrm{T}_{\mathrm{i}+\Delta \mathrm{x}}\right) /\left(\Delta x^{2} / 2 \mathrm{k}_{\mathrm{i}+\Delta \mathrm{x}}\right) \\
\mathrm{j}_{\mathrm{i}-\Delta \mathrm{x}}=\left(\mathrm{T}_{\mathrm{i}-\Delta \mathrm{x}}-\mathrm{T}_{\mathrm{i}}\right) /\left(\Delta x^{2} / 2 \mathrm{k}_{\mathrm{i}-\Delta \mathrm{x}}\right)
\end{gathered}
$$

has the form of Kirchhoff's law for the currents at a point in a circuit. Therefore, the elemental cell is composed of two current-controlled sources (connected between the nodes $\mathrm{i}-\Delta x \rightarrow \mathrm{i}$ and $\mathrm{i} \rightarrow \mathrm{i}+\Delta x)$ and a capacitor of value $\rho c_{\mathrm{e}}$.

\section{Solution of the inverse problem}

Broadly speaking, the inverse problem, to know the value of the parameters to be estimated in an instant, you must have calculated these parameters in the instant before, is a continual and iterative process composed of two loops, loop approximation (loop internal) and loop stretch (outer loop).

The "loop of stretch " is in charge of taking the whole temperature range and divide it into small sections, to go after scrolling progressively. The data required for this loop are: initial $\mathrm{T}\left(\mathrm{T}_{\text {ini }}\right)$, final $\mathrm{T}\left(\mathrm{T}_{\mathrm{end}}\right)$ and number of stretch $(\mathrm{Z})$.

Loop approximation is responsible for running PSpice go with the different values of $\mathrm{k}$, and obtain the optimal $\mathrm{k}\left(\mathrm{k}_{\mathrm{opt}}\right)$. For each approximation are performed 
three simulations $(\mathrm{k}(\mathrm{z}), \mathrm{k}(\mathrm{z})+\Delta \mathrm{k}, \mathrm{k}(\mathrm{z})-\Delta \mathrm{k})$ and take the new value of $\mathrm{k}$ that gives smallest error to assign it to $\mathrm{k}_{\mathrm{opt}}$. In each new approximation is assigned to $\mathrm{k}(\mathrm{z})$ its new value $\left(\mathrm{k}_{\mathrm{opt}}\right)$, as well as reduces the value of $\Delta \mathrm{k}_{\text {new }}=\Delta \mathrm{k} / 2$.

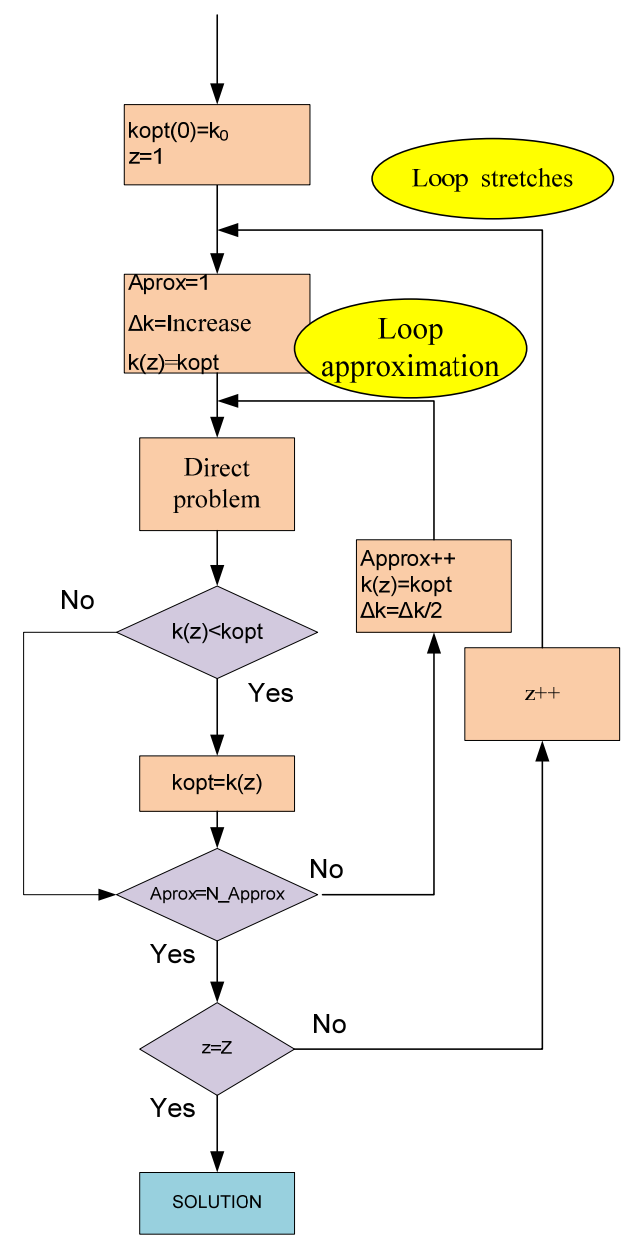

Figure 1: Inverse algorithm of resolution.

\section{Software developed}

\subsection{Introduction}

The program consists of three main windows on the first, "INICIALIZACIÓN" (Initialization), we enter the material data. In the second, "CONFIGURACIÓN" (Setup) is where you define the type of problem and all the data required for execution. Finally, the third window, "SOLUCIÓN" (Solution) is showing results. 


\subsection{INICIALIZACIÓN (Initialization)}

In the first we met (Figure 2) is the screen charge of the definition of material as well as various properties.

1. Length of body to be treated, measured in meters.

2. Density of the body to be treated, measured in $\mathrm{kg} / \mathrm{m}^{3}$.

3. Initial temperature before of the experiment in ${ }^{\circ} \mathrm{C}$.

4. Boundary conditions at the left and right side of the body.

a. Heat flux measured in $\mathrm{kW} / \mathrm{m}^{2}$.

b. Temperature measured in ${ }^{\circ} \mathrm{C}$.

c. Forced convection: Ambient temperature $\left({ }^{\circ} \mathrm{C}\right)$ and convection coefficient $\left(\mathrm{W} / \mathrm{m}^{2}{ }^{\circ} \mathrm{C}\right)$.

5. Numbers of sensors.

6. Sensor location from the left end in meters.

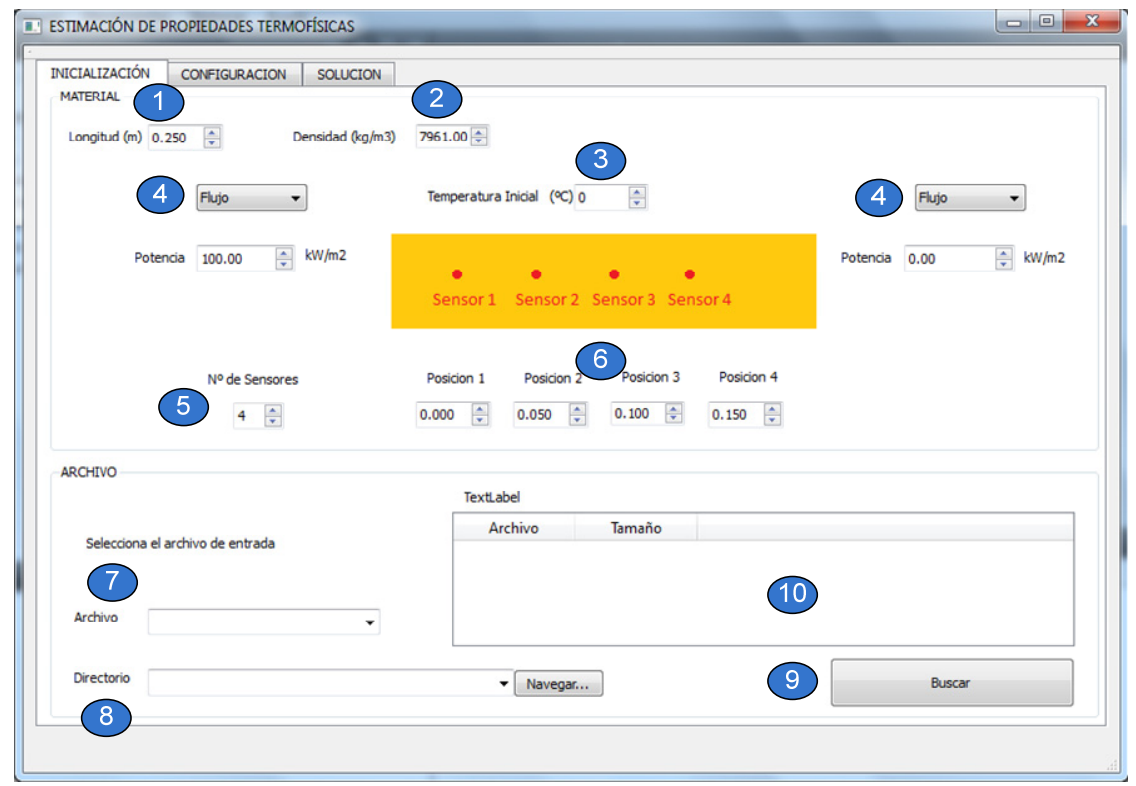

Figure 2: Initialization windows.

\section{ARCHIVO (File)}

Necessary only for cases in which one wants to solve the inverse problem or the problem of initial value.

7. Name of data file. This must be a plain text file, containing as many columns as the number of sensors for the first time, although it does not affect the process, its value is purely explanatory. Have no blank line, the first line coincides with the first measurement. You can use asterisks as wildcards. 
8. Bar directory where the browser will look for the file specified in paragraph 7.

9. Button to begin your search.

10. Window showing all files in the directory specified in section 8 and that match the name given in Section 7. Once displayed, double click on the file to be opened to check the validity of same after verification is already closed and selected.

\subsection{CONFIGURACIÓN (Setup)}

The "CONFIGURACIÓN" (Setup) windows (Figure 3) consists of a series of boxes and buttons that are activated according to the different options we have selected. We can choose between direct problem, the initial value problem and inverse problem. So we can estimate either the conductivity, such as specific heat, or both at once. To define the known variables, the program gives us a choice between two options, linear, where we give the initial and final values of the known variables, or stretches, where we can define up to nine stages.

1. Initial value of $\mathrm{k}(\mathrm{T})$.

2. $\Delta \mathrm{k}$.

3. Numbers of sections selector to define the specific heat.

4. Table to define the specific heat as a function of temperature.

5. Simulation step, must be the same as the input.

6. Initial $\mathrm{T}\left(\mathrm{T}_{\mathrm{ini}}\right)$ and final $\mathrm{T}\left(\mathrm{T}_{\mathrm{fin}}\right)$.

7. Numbers of stretches $(Z)$.

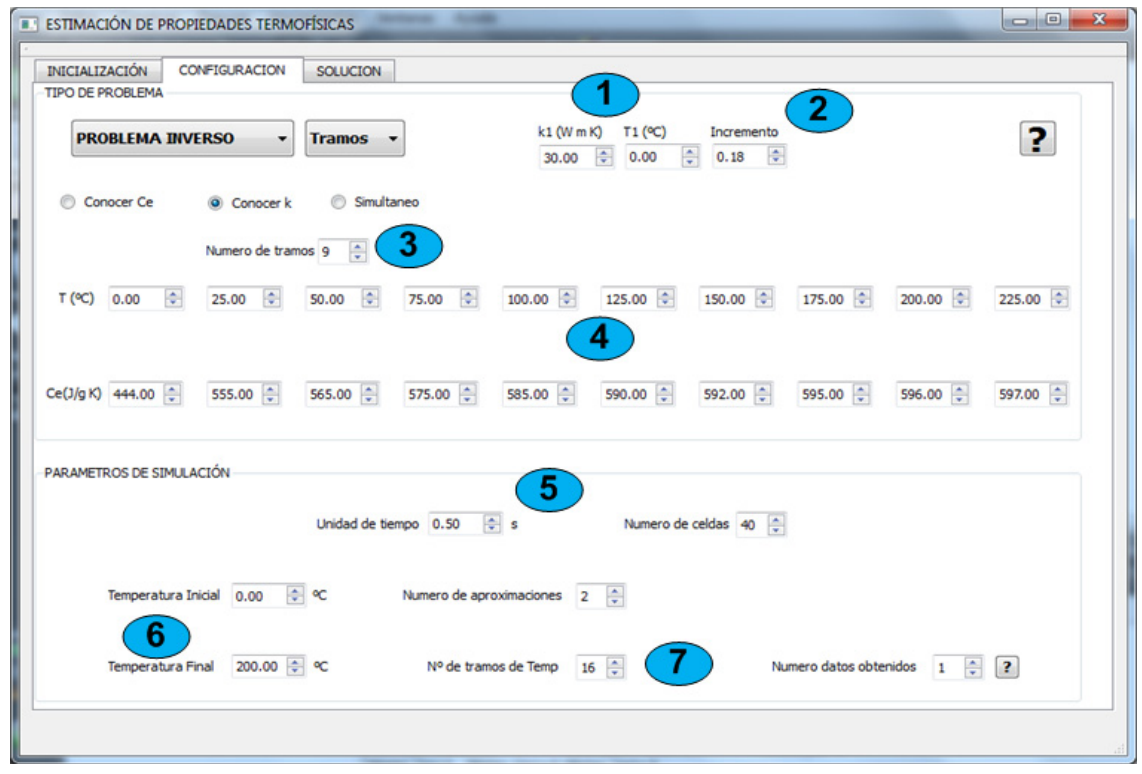

Figure 3: $\quad$ Setup window. 
Table 1: $\quad$ Properties of $2 \%$ Tungsten steel W.

\begin{tabular}{|c|c|c|c|c|c|}
\hline Temperature & $-100^{\circ} \mathrm{C}$ & $0^{\circ} \mathrm{C}$ & $100^{\circ} \mathrm{C}$ & $200^{\circ} \mathrm{C}$ & $400^{\circ} \mathrm{C}$ \\
\hline $\mathrm{k}(\mathrm{W} / \mathrm{mK})$ & ---------- & 62 & 59 & 54 & 48 \\
\hline $\mathrm{Cp}(\mathrm{kJ} / \mathrm{kgK})$ & 0.444 & 0.444 & 0.444 & 0.444 & 0.444 \\
\hline
\end{tabular}

\subsection{SOLUCION (Solution)}

In "SOLUCIÓN" (SOLUTION) window, is running the problem chosen and at the end, we show the different simulations. Placing the mouse over the graph, automatically appear calculates parameter values.

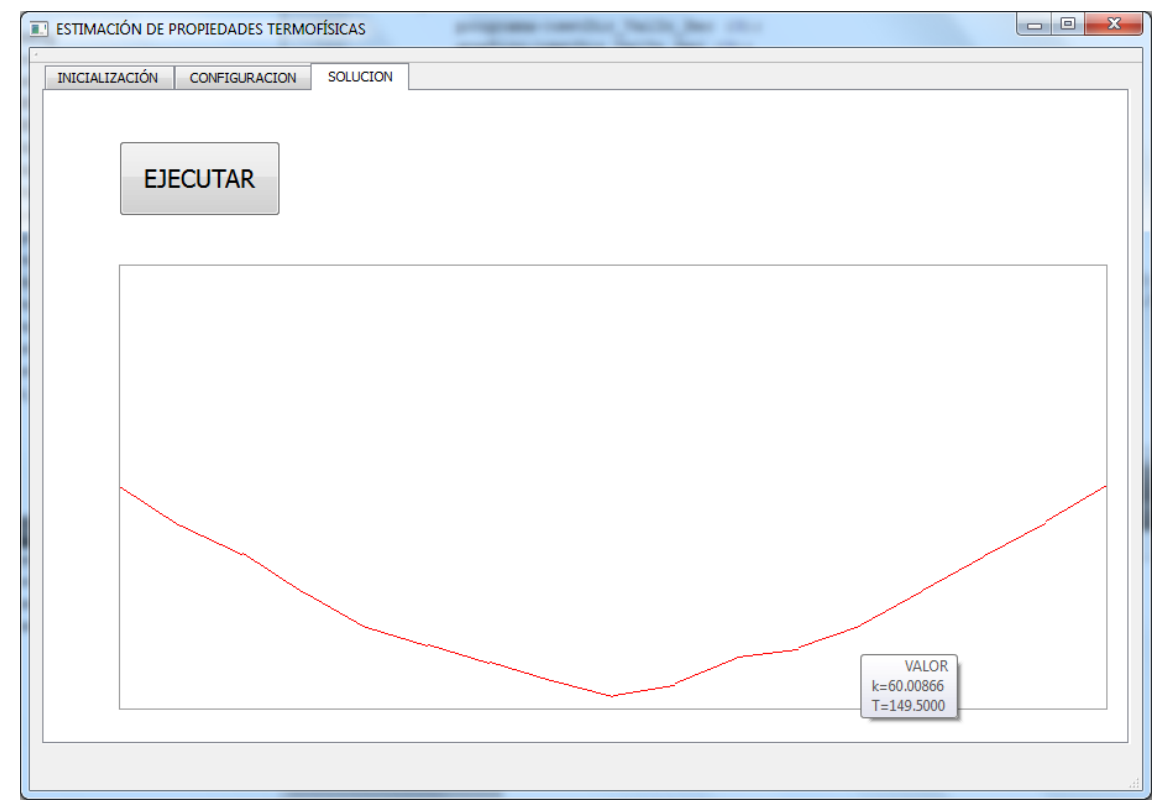

Figure 4: $\quad$ Solution window.

\section{Results}

In our case, the parameter to calculate, is the thermal conductivity (k), temperature dependent, hence the need for an iterative process. So we've taken as material for our experiment a block of " $2 \%$ Tungsten Steel W", of $0.25 \mathrm{~m}$ long. The boundary conditions for one end are a constant flow of $100 \mathrm{~W} / \mathrm{m}^{2}$ and on the rest of the contour is adiabatic.

Figure 5 shows the functional in function of the number of approximations (Z) and the increment value of $\mathrm{k}(\Delta \mathrm{k})$. we see that for very low values of $\Delta \mathrm{k}$, the program is unable to follow the dynamics of $\mathrm{k}(\mathrm{T})$, producing a very high functional results. 


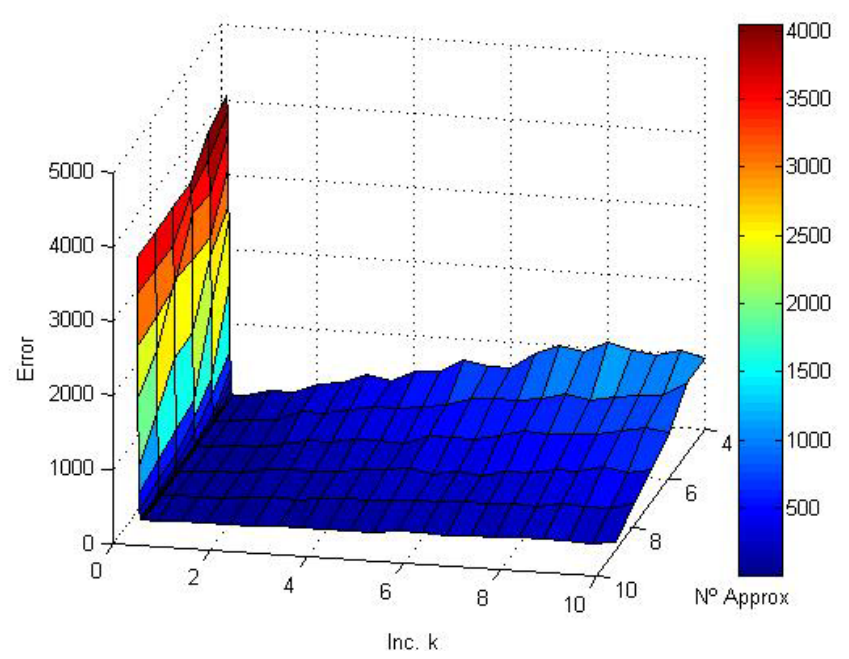

Figure 5: $\quad$ Numerical solution for the case 1.

Likewise, when $\Delta \mathrm{k}$ is very high, the program's ability to solve the problem is bigger, but its sensitivity increases, being necessary to increase the number of approximations $(Z)$ to maintain good results.

Figure 6 shows the functional as a function of the number of approximations and the initial value $\mathrm{Z}(\mathrm{k} 0)$, for $\Delta \mathrm{k}=0.4$. We see how the error decreases as we approach the initial value $\mathrm{k} 0=62$. We can also see how the errors decrease when

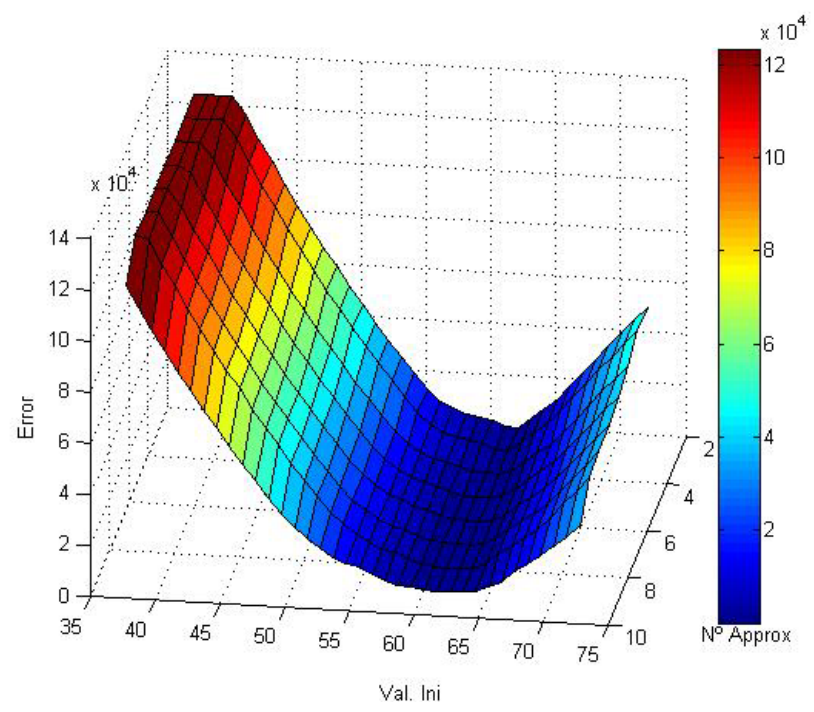

Figure 6: $\quad$ Numerical solution for the case 2. 
increases the number of approximations, because the range of variation of $\mathrm{k}$ is greater the greater the number of approximations. because the range of variation of $\mathrm{k}$ is greater the greater the number of approximations Eq. (8).

$$
\Delta k_{\text {Total }}\left(N^{\mathrm{o}} \text { Approx }\right)= \pm \sum_{n=1}^{n=N^{\circ} \text { Approx }}\left(\frac{\Delta k_{0}}{2 n}\right)
$$

\section{Conclusions}

This paper provides a methodology together with software adequate for the inverse estimation of the thermal properties temperature-dependent in a solid medium. A numerical study has been performed to identify, the thermal conductivity. In the case of the studied example, the Sequential Function Specification Method has been used together with Network Simulation Method and they appear to be efficient for the estimation. No prior information is used for the functional forms of the unknown specific heat, because this problem is considered a function estimation problem. A special device that generates a piecewise temperature-dependent function is required in conjunction with a programming routine. The use of the temperature evolution versus time measured at many positions permits an accurate estimation.

\section{Acknowledgement}

This work was supported by VI Plan Nacional de Investigación Científica, Desarrollo e Innovación Tecnológica 2008-2011, Spain, Reference ENE200912117.

\section{References}

[1] Beck, J. V., Blackwell, B., and St. Clair, C. R. Jr. Inverse heat conduction, ill-posed problems. Wiley-Interscience Pub. New York, 1985.

[2] Osman A.M. and Beck J.V. Investigation of transient heat transfer coefficients in quenching experiments. Journal Heat Transfer, 1990, 112, 843-848.

[3] Sin Kim, Min Chan Kim, Kyung Youn Kim, Non-iterative estimation of temperature-dependent thermal conductivity without internal measurements, International Journal of Heat and Mass Transfer 46 (2003) 1801-1810.

[4] Sawaf, B., Özisik, M.N., Jarny, Y., An inverse analysis to estimate linearly temperature dependent thermal conductivity components and heat capacity of an orthotropic medium, Int. Journal Heat Mass Transfer, 38, (16), 30053010, 1995.

[5] L.B. Dantas and H.R.B. Orlande, A function estimation approach for determining temperature-dependent thermophysical properties. Inverse Problem Eng., 3, 261 (1996). 
[6] Carciofi, B., Faistel, J., Aragäo G. and Laurindo, J. (2002) Determination of thermal diffusivity of mortadella using actual cooking process data. Journal of Food Engineering, 55, 89-94.

[7] Sacadura, J.F. and Osman, T.T., Emissivity estimation through the solution of an inverse heat-conduction problem. J. Thermophysics, 4, 1, 86-91 (1989).

[8] C.H. Huang and M.N. Özisik, Num. Heat Transfer A-Appl., 20, 95 (1991)

[9] Alhama F. and González-Fernández, C.F., Transient thermal behaviour of phase-change processes in solid foods with variable thermal properties, J. Food Eng., 54, 331-336, 2002.

[10] Zueco, F. Alhama F. and González-Fernández C.F., Analysis of laminar forced convection with network simulation in thermal entrance region of ducts, Int. J. Thermal Science, 43(5), 443-451, 2004.

[11] Alhama, F., Zueco, J. and González-Fernández, C.F., An inverse determination of unsteady heat fluxes using a network simulation method. J. Heat Transfer-T ASME, 125 (6), 1178-1183, 2003.

[12] Zueco, J., Alhama, F. and González-Fernández, C.F., Inverse determination of the specific heat of foods, J. Food Eng., 64 (3), 347-353, 2004. 